Stud. Univ. Babeş-Bolyai Math. 65(2020), No. 4, 495-502

DOI: $10.24193 /$ subbmath.2020.4.02

\title{
On the stability of solutions of fractional non conformable differential equations
}

\author{
Paulo M. Guzmán, Luciano M. Lugo Motta Bittencurt and \\ Juan E. Nápoles Valdes
}

\begin{abstract}
In this note we obtain sufficient conditions under which we can guarantee the stability of solutions of a fractional differential equations of non conformable type and we obtain some fractional analogous theorems of the direct Lyapunov method for a given class of equations of motion.
\end{abstract}

Mathematics Subject Classification (2010): 34A08.

Keywords: Fractional non conformable system of equations, Lyapunov second method, stability, asymptotic stability, instability.

\section{Introduction}

Fractional calculus concerns the generalization of differentiation and integration to non-integer (fractional) orders. The subject has a long mathematical history being discussed for the first time already in the correspondence of Leibniz with L'Hopital when this replied "What does $\frac{d^{n}}{d x^{n}} f(x)$ mean if $n=\frac{1}{2}$ ?" in September 30 of 1695 . Over the centuries many mathematicians have built up a large body of mathematical knowledge on fractional integrals and derivatives. Although fractional calculus is a natural generalization of calculus, and although its mathematical history is equally long, it has, until recently, played a negligible role in physics. One reason could be that, until recently, the basic facts were not readily accessible even in the mathematical literature (see [13]). The nature of many systems makes that they can be more precisely modeled using fractional differential equations. The differentiation and integration of arbitrary orders have found applications in diverse fields of science and engineering like viscoelasticity, electrochemistry, diffusion processes, control theory, heat conduction, electricity, mechanics, chaos, and fractals (see [5], [6] and [13]). Lyapunov's Second or Direct Method is unique in that it does not require a characterization of the solutions to determine stability. This method often allows us to determine whether a differential equation is stable without knowing anything about what the solutions look like, so it 
is ideal for dealing with nonlinear systems. The method uses a supplementary function called a Lyapunov function to determine properties of the asymptotic behavior of solutions of a differential equation. It is known that the method of Lyapunov functions is a tool used in the analysis of stability, in many classes of differential equations of disturbed movement, so it is interesting to investigate an extension of the method for non-integer order systems (see [9] and [10] and bibliography there). Such extension is based on the concept of a local fractional derivative non conformable, defined by the authors in a previous paper (see [2]) which is presented below. In this paper the application of a fractional-like derivative of the Lyapunov function for the stability analysis of solutions of the equations of perturbed motion with a fractional-like derivative of the state vector is discussed. Some fractional analogous theorems of the direct Lyapunov method for a given class of equations of motion are presented.

\section{Preliminary results}

It is necessary to present some necessary definitions for our work. Be $\alpha \in(0,1]$ and define a continuous function $f:\left[t_{0},+\infty\right) \rightarrow \mathbb{R}$.

First, let's remember the definition of $N_{1}^{\alpha} f(t)$, a non conformable fractional derivative of a function in a point $t$ defined in [9] and that is the basis of our results, that are close resemblance of those found in classical qualitative theory.

Definition 2.1. Given a function $f:\left[t_{0},+\infty\right) \rightarrow \mathbb{R}, t_{0}>0$. Then the $\mathrm{N}$-derivative of $f$ of order $\alpha$ is defined by

$$
N_{1}^{\alpha} f(t)=\lim _{\varepsilon \rightarrow 0} \frac{f\left(t+\varepsilon e^{t^{-\alpha}}\right)-f(t)}{\varepsilon}
$$

for all $t>0, \alpha \in(0,1)$. If $\mathrm{f}$ is $\alpha$-differentiable in some $(0, a)$, and $\lim _{t \rightarrow 0^{+}} N_{1}^{(\alpha)} f(t)$ exists, then define $N_{1}^{(\alpha)} f(0)=\lim _{t \rightarrow 0^{+}} N_{1}^{(\alpha)} f(t)$.

If the $\mathrm{N}$-derivative of the function $x(t)$ of order $\alpha$ exists and is finite in $\left(t_{0}, \infty\right)$, we will say that $x(t)$ is $\mathrm{N}$-differentiable in $I=\left(t_{0}, \infty\right)$.

Remark 2.2. The use in definition 2.1 of the limit of a certain incremental quotient, instead of the integral used in the classical definitions of fractional derivatives, allows us to give the following interpretation of the N-derivative. Suppose that the point moves in a straight line in $\mathbb{R}_{+}$. For the moments $t_{1}=t$ and $t_{2}=t+h e^{t^{-\alpha}}$ where $h>0$ and $\alpha \in(0,1]$ and we denote $S\left(t_{1}\right)$ and $S\left(t_{2}\right)$ the path traveled by point $\mathrm{P}$ at time $t_{1}$ and $t_{2}$ so we have

$$
\frac{S\left(t_{2}\right)-S\left(t_{1}\right)}{t_{2}-t_{1}}=\frac{S\left(t+h e^{t^{-\alpha}}\right)-S(t)}{h e^{t^{-\alpha}}}
$$

this is the average $\mathrm{N}$-speed of point $\mathrm{P}$ over time $h e^{t^{-\alpha}}$. Let's consider

$$
\operatorname{Lim}_{h \rightarrow 0} \frac{S\left(t+h e^{t^{-\alpha}}\right)-S(t)}{h e^{t^{-\alpha}}} .
$$


When $\alpha=1$, this is the usual instantaneous velocity of a point $\mathrm{P}$ at any time $t>0$. If $\alpha \in(0,1)$ this is the instantaneous $\mathrm{q}$-speed of the point $\mathrm{P}$ for any $t>0$. Therefore, the physical meaning of the $\mathrm{N}$-derivative is the instantaneous q-change rate of the state vector of the considered mechanics or another nature of the system.

Remark 2.3. The N-derivative solves almost all the insufficiencies that are indicated to the classical fractional derivatives. In particular we have the following result.

Theorem 2.4. (See [2]) Let $f$ and $g$ be $N$-differentiable at a point $t>0$ and $\alpha \in(0,1]$. Then

a) $N_{1}^{\alpha}(a f+b g)(t)=a N_{1}^{\alpha}(f)(t)+b N_{1}^{\alpha}(g)(t)$.

b) $N_{1}^{\alpha}\left(t^{p}\right)=e^{t^{-\alpha}} p t^{p-1}, p \in \mathbb{R}$.

c) $N_{1}^{\alpha}(\lambda)=0, \lambda \in \mathbb{R}$.

d) $N_{1}^{\alpha}(f g)(t)=f N_{1}^{\alpha}(g)(t)+g N_{1}^{\alpha}(f)(t)$.

e) $N_{1}^{\alpha}\left(\frac{f}{g}\right)(t)=\frac{g N_{1}^{\alpha}(f)(t)-f N_{1}^{\alpha}(g)(t)}{g^{2}(t)}$.

f) If, in addition, $f$ is differentiable then $N_{1}^{\alpha}(f)=e^{t^{-\alpha}} f^{\prime}(t)$.

g) Being $f$ differentiable and $\alpha=n$ integer, we have $N_{1}^{n}(f)(t)=e^{t^{-n}} f^{\prime}(t)$.

Remark 2.5. The relations a), c), d) and e) are similar to the classical results mathematical analysis, these relationships are not established (or do not occur) for fractional derivatives of global character (see [5] and [13] and bibliography there). The relation c) is maintained for the fractional derivative of Caputo. Cases c), f) and g) are typical of this non conformable local fractional derivative.

Now we will present the equivalent result, for $N_{1}^{\alpha}$, of the well-known chain rule of classic calculus and that is basic in the Second Method of Lyapunov, for the study of stability of perturbed motion.

Theorem 2.6. (See [2]) Let $\alpha \in(0,1], g N$-differentiable at $t>0$ and $f$ differentiable at $g(t)$ then

$$
N_{1}^{\alpha}(f \circ g)(t)=f^{\prime}(g(t)) N_{1}^{\alpha} g(t) .
$$

Definition 2.7. The non conformable fractional integral of order $\alpha$ is defined by the expression

$$
{ }_{N} J_{t_{0}}^{\alpha} f(t)=\int_{t_{0}}^{t} \frac{f(s)}{e^{s^{-\alpha}}} d s .
$$

The following statement is analogous to the one known from the Ordinary Calculus.

Theorem 2.8. Let $f$ be $N$-differentiable function in $\left(t_{0}, \infty\right)$ with $\alpha \in(0,1]$. Then for all $t>t_{0}$ we have

a) If $f$ is differentiable ${ }_{N} J_{t_{0}}^{\alpha}\left(N_{1}^{\alpha} f(t)\right)=f(t)-f\left(t_{0}\right)$.

b) $N_{1}^{\alpha}\left({ }_{N} J_{t_{0}}^{\alpha} f(t)\right)=f(t)$.

Proof. a) From definition we have

$$
{ }_{N} J_{t_{0}}^{\alpha}\left(N_{1}^{\alpha} f(t)\right)=\int_{t_{0}}^{t} \frac{N_{1}^{\alpha} f(s)}{e^{s^{-\alpha}}} d s=\int_{t_{0}}^{t} \frac{f^{\prime}(s) e^{s^{-\alpha}}}{e^{s^{-\alpha}}} d s=f(t)-f\left(t_{0}\right) .
$$


b) Analogously we have

$$
N_{1}^{\alpha}\left({ }_{N} J_{t_{0}}^{\alpha} f(t)\right)=e^{t^{-\alpha}} \frac{d}{d t}\left[\int_{t_{0}}^{t} \frac{f(s)}{e^{s^{-\alpha}}} d s\right]=f(t) .
$$

\section{N-derivative of the Lyapunov function and conditions of stability and instability of movement}

Consider the following system of fractional N-differential equations

$$
\begin{gathered}
N_{1}^{\alpha} x(t)=f(t, x(t)), \\
x\left(t_{0}\right)=x_{0} .
\end{gathered}
$$

where $x \in \mathbb{R}^{n}, f \in C\left(\mathbb{R}_{+} \times \mathbb{R}^{n}, \mathbb{R}^{n}\right), t_{0}>0$. It is further assumed that for $\left(t_{0}, x_{0}\right) \in$ $\operatorname{int}\left(\mathbb{R}_{+} \times \mathbb{R}^{n}\right)$ the initial value problem (3.1-3.2) has a solution $x(t) \in C^{\alpha}(I)$ for all $t>t_{0}>0$. In addition, it is assumed that $f(t, 0)=0$ for all $t>t_{0}>0$.

Let for equation (3.1) a Lyapunov-type function $V(t, x) \in C^{\alpha}\left(I \times \mathbb{R}^{n}\right)$ be constructed in some way such that $V(t, 0)=0$ for all $t>0$. Introduce the notation $S_{r}=\left\{x \in \mathbb{R}^{n}:\|x\|<r, r>0\right\}$.

Definition 3.1. Let $V$ be a continuous and $\alpha$-differentiable function (scalar or vector), $V: I \times S_{r} \rightarrow \mathbb{R}^{p}(p=1$ or $p=m$, respectively), and $x(t)$ be the solution of the IVP (3.1-3.2), which exists and is defined on $I \times S_{r}$. Corresponding to $\mathrm{V}(\mathrm{t}, \mathrm{x})$ we define for $(t, x) \in I \times S_{r}$ the function

$$
+N_{(3.1)}^{\alpha} V(t, x)=\lim _{h \rightarrow 0} \frac{\left[V\left(t+h, x+h^{\alpha} f(t, x)\right)-V(t, x)\right]}{h}
$$

is the N-derivative of $V(t, x)$ with respect to the system (3.1) (or along the solutions of system (3.1)).

We will now present the results analogous to those known from the Second Method of Lyapunov, for the study of the stability of systems (3.1).

With $C(\mathbb{R})$ and $C I(\mathbb{R})$ we respectively denote the families of continuous functions and increasing continuous functions defined on $\mathbb{R}$.

Definition 3.2. (see [11]). $C S(\mathbb{R})=\{h \in C(\mathbb{R}): x h(x)>0, x \neq 0\}$

Definition 3.3. (see $[11])$. $C C(\mathbb{R}):=C I(\mathbb{R}) \cap C S(\mathbb{R})$.

Definition 3.4. A continuous function $\beta:[0, t) \rightarrow[0,+\infty)$ is said to belong to class-K if it is strictly increasing and $\beta(0)=0$.

Theorem 3.5. Suppose that for the system (3.1) there is a function $N$-differentiable $V(t, x)$ and the functions $a, b \in K$, such that

i) $V(t, x) \geq a(\|x\|)$,

ii) $V(t, x) \leq b(\|x\|)$, and

$$
+N_{(3.1)}^{\alpha} V(t, x) \leq 0,
$$

for all $(t, x) \in I \times S_{r}$. Then the solution $x=0$ of the system (3.1) is uniformly stable. 
Proof. Let $x(t)$ the solution of system (3.1) which satisfies the initial condition $\left(t_{0}, x_{0}\right) \in I \times S_{r}$ and that exists for all $t \geq t_{0}$. Let $t_{0} \in I$ and $0<\varepsilon<r$. Under conditions i), ii) of the theorem let's choose $\delta=\delta(\varepsilon)>0$ such that

$$
b(\delta)<a(\varepsilon)
$$

Let's prove that if $\left\|x_{0}\right\|<\delta$ then $\|x(t)\|<\varepsilon$ for all $t \geq t_{0}$. If this were not true, then there is a solution $x=x(t)$ such that for $\left\|x_{0}\right\|<\delta$ there exists $t_{1}>t_{0}$ what satisfies $\left\|x\left(t_{1}\right)\right\|=\varepsilon$, and $\|x(t)\|<\varepsilon$ for all $t \in\left[t_{0}, t_{1}\right)$.

Under Theorem 8 and condition (3.2), we have

$$
V(t, x(t))-V\left(t_{0}, x_{0}\right)={ }_{N} J_{t_{0}}^{\alpha}\left(N_{1}^{\alpha} V(t, x(t))\right)
$$

and so

$$
V(t, x(t))-V\left(t_{0}, x_{0}\right) \leq 0
$$

of this last inequality for $t=t_{1}$ we get

$$
a(\varepsilon) \leq V\left(t_{1}, x\left(t_{1}\right)\right) \leq V\left(t_{0}, x_{0}\right) \leq b(\|x\|)<a(\varepsilon)
$$

The resulting inequality is evidently false. This proves Theorem (3.5).

Next, we present the conditions that guarantee the asymptotic stability of the null solution of the fractional system (3.1).

Theorem 3.6. In addition to the conditions i)-ii) of the previous theorem, suppose that instead of condition (3.4), we have

$$
+N_{(3.1)}^{\alpha} V(t, x) \leq-c(\|x\|),
$$

for all $(t, x) \in I \times S_{r}$ and $c$ is a function of class $K$. Then the solution $x=0$ of the system (3.1) is uniform asymptotically stable.

Proof. Under the conditions of the theorem, the solution $x=0$ of the system (3.1) is uniformly stable since the conditions of the previous theorem are satisfied. We show that this solution is uniformly asymptotically stable.

Let $0<\varepsilon<r$ and $\delta=\delta(\varepsilon)>0$ as before. For $\varepsilon_{0} \leq r$ let's choose $\delta_{0}=\delta_{0}\left(\varepsilon_{0}\right)>0$ and we consider the solution $x(t)$ with initial conditions $t_{0} \in I$ and $\left\|x_{0}\right\|<\delta_{0}$. For $t_{0}<t \leq t_{0}+T(\varepsilon)$, where $T(\varepsilon)$ will be defined by an implicit expression that will be specified later, such a solution satisfies $\|x(t)\| \geq \delta(\varepsilon)$. Let's prove that under the conditions of the theorem this is impossible. From (3.8) and Theorem 2.8 we obtain

$$
\begin{aligned}
& V(t, x(t))-V\left(t_{0}, x_{0}\right)={ }_{N} J_{t_{0}}^{\alpha}\left(N_{1}^{\alpha} V(t, x(t))\right) \leq-{ }_{N} J_{t_{0}}^{\alpha}(c(\|x(t)\|)) \\
& V(t, x(t))-V\left(t_{0}, x_{0}\right) \leq-\int_{t_{0}}^{t} \frac{c(\|x(t)\|)}{e^{\left(s-t_{0}\right)^{-\alpha}}} d s .
\end{aligned}
$$

We denote by

so we have from $(3.9)$

$$
{ }_{N} J_{t_{0}}^{\alpha}(e)=\int_{t_{0}}^{t} \frac{d s}{e^{\left(s-t_{0}\right)^{-\alpha}}}=E(t)-E\left(t_{0}\right),
$$

$$
V(t, x(t)) \leq b\left(\delta_{0}\right)-c(\delta(\epsilon))_{N} J_{t_{0}}^{\alpha}(e)
$$


For $t=t_{0}+T(\varepsilon)$ the inequality (3.10) we can write it as

$$
0<a(\delta(\varepsilon)) \leq V\left(t_{0}+T(\varepsilon), x\left(t_{0}+T(\varepsilon)\right)\right) \leq b\left(\delta_{0}\right)-c(\delta(\varepsilon))[E(T(\varepsilon))] \leq 0 .
$$

This contradiction shows that there is $t_{1} \in\left[t_{0}, t_{0}+T(\varepsilon)\right]$ for which $\left\|x\left(t_{1}\right)\right\|<\delta(\varepsilon)$. Therefore, the estimate $\|x(t)\|<\varepsilon$ is true for all $t \geq t_{0}+T(\varepsilon)$ as $\left\|x_{0}\right\|<\delta_{0}$ and $\operatorname{Lim}_{t \rightarrow \infty}\|x(t)\|=0$ uniformly in $t_{0} \in I$. This proves Theorem.

Next, we will establish the conditions for the instability of the solution $x=0$ of the system (3.1).

Theorem 3.7. Suppose that for the system (3.1) there is an $N$-differentiable Lyapunov function $V(t, x)$ such that on $I \times B_{q}$ with $B_{q} \subset B_{\varepsilon}$ satisfies the assumptions

i) $0<V(t, x) \leq b(\|x\|)$,

ii) ${ }_{+} N_{(3.1)}^{\alpha} V(t, x) \leq \lambda V(t, x)+W(t, x)$, with $\lambda>0$ and $V: I \times B_{q} \rightarrow \mathbb{R}_{+}$, $W(t, x) \geq 0$

iii) the solution $x=0$ belongs to $\delta B_{q}$;

iv) $V(t, x)=0$ on $I \times\left(\delta B_{q} \cap B_{\varepsilon}\right)$.

Then the solution $x=0$ is unstable.

Proof. From assumptions ii), and Theorem 2.8 we have

$$
V(t, x(t)) \geq V\left(t_{0}, x\left(t_{0}\right)\right) \exp \left[\lambda_{N} J_{t_{0}}^{\alpha}(e)\right], t \geq t_{0},
$$

Let the solution with initial condition $x_{0} \in N$ be a neighborhood of $x=0$. So that for any $t \geq t_{0}$ satisfying the estimate (3.11) along the solution $x(t)$, then it is clear that for $t \rightarrow \infty$, the function $V(t, x(t))$ grows indefinitely, whereas under the conditions of Theorem 3.5 is bounded. Therefore, for the solution $x(t)$ there exists $t^{\prime}$ such that $x\left(t^{\prime}\right)$ will leave the region $B_{\varepsilon}$. This shows the instability of the solution $x=0$ of the system (3.1), which proves the theorem.

Example 3.8. We consider the Lienard N-fractional system

$$
\left\{\begin{array}{l}
N_{1}^{\alpha} x(t)=y-F(t) \\
N_{1}^{\alpha} y(t)=-g(x(t))
\end{array}\right.
$$

with

$$
F(x)=\int_{0}^{x} f(r) d r
$$

and we take the Lyapunov Function

$$
V(t, x, y)=\frac{y^{2}}{2}+G(x)
$$

with

$$
G(x)=\int_{0}^{x} g(s) d s .
$$

Under assumptions on the continuous functions $f$ and $g$ :

1. $f(x)>0$ for all $x \in \mathbb{R}$,

2. $x g(x)>0$ for all $x \neq 0$, 
we have the stability of solution $x=y=0$ of system (3.12). From (3.13) we have

$$
{ }_{+} N_{(3.1)}^{\alpha} V(t, x, y)=-g(x) F(x) \leq 0 .
$$

By virtue of Theorem 3.5 the desired result is obtained.

\section{References}

[1] Abdeljawad, T., On conformable fractional calculus, Journal of Computational and Applied Mathematics, 279(2015), 57-66.

[2] Guzmán, P.M., Langton, G., Lugo Motta, L., Medina, J., Nápoles V., J.E., A New definition of a fractional derivative of local type, J. Mathem. Anal., 9(2018), no. 2, 8898.

[3] Guzmán, P.M., Lugo Motta, L., Nápoles V., J.E., A note on stability of certain Lienard fractional equation, International Journal of Mathematics and Computer Science, 14(2019), no. 2, 301-315.

[4] Khalil, R., Al Horani, M., Yousef, A., Sababheh, M., A new definition of fractional derivative, Journal of Computational and Applied Mathematics, 264, 65-70.

[5] Kilbas, A., Srivastava, M.H., Trujillo, J.J., Theory and Application on Fractional Differential Equations, vol. 204, North-Holland Mathematics Studies, 2006.

[6] Lakshmikantham, V., Leela, S., Devi, J.V., Theory of Fractional Dynamic Systems, Cambridge: Cambridge Scientific Publ., 2009.

[7] Liénard, A., Étude des oscillations entretenues, Revue Génerale de l'Électricité, 23 (1928), 901-912, 946-954.

[8] Lyapunov, A.M., The General Problem of Motion Stability, (in Russian), Leningrad, Moscow: ONTI, 1935.

[9] Martynyuk, A.A., On the stability of a system of equations with fractional derivatives with respect to two measures, Journal of Mathematical Sciences, 217(2016), no. 4, 468475.

[10] Martynyuk, A.A., Lyapunov direct method, stability, asymptotic stability, instability, Dopov. Nats. Akad. Nauk Ukr., (2018), no. 6, 9-16.

[11] Nápoles V., J.E., A note on the asymptotic stability in the whole of nonautonomous systems, Revista Colombiana de Matemáticas, 33(1999), 1-8.

[12] Nápoles V., J.E., Guzman, P.M., Lugo Motta, L., Some new results on the non conformable fractional calculus, Advances in Dynamical Systems and Applications, 13(2018), no. 2, 167-175.

[13] Podlybny, I., Fractional Differential Equations, London, Acad. Press, 1999.

Paulo M. Guzmán

Universidad Nacional del Nordeste, FaCENA, Av. Libertad 5470,

3400 - Corrientes Capital, Argentina

e-mail: pguzman@exa.unne.edu.ar

Universidad Nacional del Nordeste, Facultad de Ciencias Agrarias, Sargento Cabral 2131, 3400 - Corrientes Capital, Argentina 
502 P.M. Guzmán, L.M. Lugo Motta Bittencurt and J.E. Nápoles Valdes

Luciano M. Lugo Motta Bittencurt

Universidad Nacional del Nordeste, FaCENA, Av. Libertad 5470, 3400 - Corrientes Capital, Argentina

e-mail: lmlmb@yahoo.com

Juan E. Nápoles Valdes

Universidad Nacional del Nordeste, FaCENA, Av. Libertad 5470, 3400 - Corrientes Capital, Argentina

e-mail: jnapoles@exa.unne.edu.ar

Universidad Tecnológica Nacional, FRRe,

French 414, 3500 - Resistencia - Chaco, Argentina 